\title{
The Concept of Hyoid Posture
}

\author{
Rebecca Z. German • Regina Campbell-Malone • \\ A. W. Crompton · Peng Ding • Shaina Holman • \\ Nicolai Konow $\cdot$ Allan J. Thexton
}

Published online: 27 March 2011

(C) Springer Science+Business Media, LLC 2011

In their article "Evaluating the Structural Properties of Suprahyoid Muscles and their Potential for Moving the Hyoid," Pearson et al. [1] present the results of calculations based upon measurements from anatomical dissections to provide a background for understanding the power and work that potentially can be generated by selected suprahyoid muscles. As they clearly point out, such results are useful only in the context of specific additional physiological information.

What that physiological context is and how one should interpret their statement that "muscle function is limited by structural data" are not made clear. The information necessary to understand normal and, hence, abnormal hyoid movement is scattered across many papers and a myriad of subdisciplines. Our goal is to point to some of the disparate sources of information that are relevant to hyoid movement, including the Pearson et al. article, in order to generate a better understanding of the wide variety of factors that may affect hyoid movement.

Hyoid movement is critical to swallowing, mastication, intraoral transport, vocalization, and respiration. Equally

R. Z. German $(\varangle) \cdot$ R. Campbell-Malone $\cdot$ P. Ding · S. Holman Department of Physical Medicine and Rehabilitation, Johns Hopkins University, Baltimore, MD 21287, USA e-mail: rzgerman@jhu.edu

\author{
A. W. Crompton \\ Museum of Comparative Zoology, Harvard University, \\ Cambridge, MA 01238, USA
}

N. Konow

Department of Ecology and Evolutionary Biology,

Brown University, Providence, RI 02912, USA

A. J. Thexton

King's College London, London SE1 7EH, UK important for some of those behaviors is non-movement, or stabilization, of the hyoid. "Hyoid posture" is the concept that the position of the hyoid and the dynamic maintenance of that position is critical to function. Airway patency, some oral functions and speech are examples of when constancy of position is critical. Because the hyoid is a "floating bone" and its position is maintained by a sling of muscles, some of which do not articulate with another fixed bone (e.g., middle pharyngeal constrictor, hyoglossus), the limits and capabilities of the hyoid musculature are essential for hyoid posture.

The paucity of information on several aspects of hyoid muscle contraction contributes to the difficulty of predicting hyoid behavior from the anatomy of its muscles. One problem is the lack of basic information on the lengthtension and force-velocity characteristics of individual hyoid muscles. For several functions, the anterior and posterior suprahyoid and infrahyoid muscles are active simultaneously [2-6]. Such simultaneous activities of agonist and antagonist muscles are important for stabilization and precise trajectories of movement [7]. Excitation patterns also vary tremendously within the hyoid muscles $[2,4,8]$. Multiple fine-wire or micropatch electrodes in geniohyoid and mylohyoid have demonstrated that the patterns of EMG activity varied not only with the specific function (suck, swallow) but also with the intramuscular site within the individual muscles.

The relationship between EMG activity and muscle strain, or length change, is also complex. The differences in kinematics that result from concentric (muscle shortening), isometric (static length), and eccentric (muscle lengthening) contractions suggest that knowledge of the anatomical properties of a muscle, including its origin, insertion, fiber pennation and effective fiber population, is still not sufficient to permit prediction of the movements that result from its activation. Furthermore, it is entirely possible that 
different portions of a muscle can generate different contraction patterns [9, 10]. Recent work [11] also shows that significant regionalization of function exists in geniohyoid and sternohyoid during a simple reflex behavior.

While factors such as fatigue resistance, muscle fiber twitch times, and tetanic fusion frequency have been investigated with respect to specific, even isolated, hyoidrelated muscles in a few species $[5,6]$, there is a general lack of such information for the hyoid musculature as a whole and a serious deficiency of information in the case of man. There are also a number of other considerations that have yet to be adequately investigated, such as elastic compliance and hysteresis in the hyoid musculature and the extent to which successive positions of the hyoid are determined by passive equilibria or actively by positional sensory feedback to the motoneuron pools. The investigation of the latter is complicated by the apparent paucity and uncertain nature of the sensory receptors in those muscles. At the very least, in order to gain a very basic understanding of hyoid movement, the EMG and kinetic activity of the sets of agonist and antagonist muscles such as geniohyoid, mylohyoid, sternohyoid, and stylohyoid should be considered simultaneously with craniocervical posture.

Such studies may be problematic in man. There is also a potential problem in interpreting EMG activity recorded from hyoid muscles in humans using conventional surface electrodes. Conventional electrodes detect signals from a volume that is likely to contain not only different hyoid muscles but different motor unit populations within those muscles. The functional heterogeneity that has already been identified among the motor units of hyoid muscles by other methods $[4,9,12,13]$ consequently is unlikely to be detected. If the goal of a study was to detect, within an EMG recording, signs of a particular neurological deficit, conventional surface electrode recording methods could mask the specifics of that pathophysiology.

Pearson et al. [1] conclude that the geniohyoid "has the most potential to displace the hyoid in an anterior direction" but do acknowledge that additional information on "fiber types, passive forces ... and motor units recruited for a specific task ... are essential to understanding function." The lack of information on the level of recruitment of geniohyoid motor units to move the hyoid forward does, however, tend to undermine the use of physiological crosssectional area (PCSA) as a predictor of forward pull on the hyoid, although the PCSA may well indicate the upper limit of that pull. Before firm conclusions can be drawn, it is necessary to obtain detailed in vivo data on both the hyoid kinematics and the distribution of the intramuscular electromyographic activity simultaneously within the individual muscles that make up the three-way lines of action on the hyoid.

\section{References}

1. Pearson WG Jr, Langmore SE, Zumwalt AC. Evaluating the structural properties of suprahyoid muscles and their potential for moving the hyoid. Dysphagia. 2010 Nov 11 [Epub ahead of print]. doi:10.1007/s00455-010-9315-z.

2. German RZ, Crompton AW, Thexton AJ. Integration of the reflex pharyngeal swallow into rhythmic oral activity in a neurologically intact pig model. J Neurophysiol. 2009;102(2):1017-25.

3. Perlman A, Palmer P, McCulloch T, Van Daele D. Electromyographic activity from human laryngeal, pharyngeal and submental muscles during swallowing. J Appl Physiol. 1999;86(5):1663-9.

4. Thexton AJ, Crompton AW, German RZ. Electromyographic activity during the reflex pharyngeal swallow in the pig: Doty and Bosma (1956) revisited. J Appl Physiol. 2007;102(2):587-600.

5. van Lunteren E, Haxhiu MA, Cherniack NS. Mechanical function of hyoid muscles during spontaneous breathing in cats. J Appl Physiol. 1987;62:582-90.

6. van Lunteren E, Manubay P. Contractile properties of feline genioglossus, sternohyoid, and sternothyroid muscles. J Appl Physiol. 1992;72(3):1010-5.

7. Özkaya N, Nordin M. Fundamentals of biomechanics: equilibrium, motion, and deformation. New York: Springer; 1999.

8. Thexton AJ, Crompton AW, Owerkowicz T, German RZ. Impact of rhythmic oral activity on the timing of muscle activation in the swallow of the decerebrate pig. J Neurophysiol. 2009;101(3): 1386-93.

9. Konow N, Crompton AW, Thexton AJ, German RZ. Regional differences in length change and electromyographic heterogeneity in sternohyoid muscle during infant mammalian swallowing. J Appl Physiol. 2010;109:439-48.

10. Lapatki BG, Oostenveld R, Van Dijk JP, Jonas IE, Zwarts MJ, Stegeman DF. Topographical characteristics of motor units of the lower facial musculature revealed by means of high-density surface EMG. J Neurophysiol. 2006;95(1):342-54.

11. Wentzel SE, Konow N, German RZ. Regional differences in hyoid muscle activity and length dynamics during mammalian head shaking. J Exp Zool A Ecol Genet Physiol. 2011;315(3): 111-20. doi:10.1002/jez.655.

12. Tsuiki S, Ono T, Ishiwata Y, Kuroda T. Functional divergence of human genioglossus motor units with respiratory-related activity. Eur Respir J. 2000;15(5):906-10.

13. van Lunteren E, Dick TE. Heterogeneity within geniohyoid motor unit subpopulations in firing patterns during breathing. Respir Physiol. 2001;124(1):23-33.

Rebecca Z. German $\mathrm{PhD}$

Regina Campbell-Malone $\mathrm{PhD}$

A. W. Crompton $\mathrm{PhD}$

Peng Ding $\mathrm{MD}, \mathrm{PhD}$

Shaina Holman BA

Nicolai Konow $\mathrm{PhD}$

Allan J. Thexton PhD 